\title{
Wnt7a promotes the osteogenic differentiation of human mesenchymal stem cells
}

\author{
LEILUO YANG ${ }^{1,2}$, QING LI ${ }^{2}$, JUNHONG ZHANG ${ }^{3}$, PENGCHENG LI ${ }^{4}$, PINGJIANG AN ${ }^{2}$,

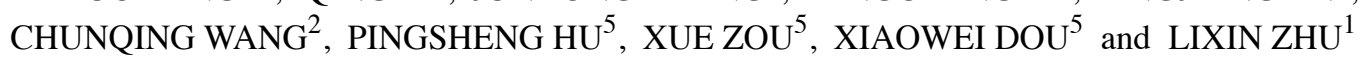

\author{
${ }^{1}$ Department of Spinal Surgery, Orthopaedic Medical Center, Zhujiang Hospital, Southern Medical University, \\ Guangzhou, Guangdong 510282; ${ }^{2}$ Department of Orthopedics, The Affiliated Hospital of Guizhou Medical University, \\ Guiyang, Guizhou 550001; ${ }^{3}$ Department of Pathology, Hebei Eye Hospital, Xingtai, Hebei 054000; \\ ${ }^{4}$ Department of Burns and Plastic Surgery, The 8th Medical Center of Chinese PLA General Hospital, Beijing 100091; \\ ${ }^{5}$ Clinical Research Center, The Affiliated Hospital of Guizhou Medical University, Guiyang, Guizhou 550001, P.R. China
}

Received March 21, 2020; Accepted March 11, 2021

DOI: $10.3892 /$ ijmm.2021.4927

\begin{abstract}
Mesenchymal stem cells (MSCs) have the ability of differentiating into osteoblasts. Elucidating the molecular mechanisms of MSC differentiation into osteoblasts may provide novel therapeutic strategies for bone-related diseases. Increasing evidence has confirmed that Wnt signaling plays the key role in osteoblast differentiation; however, the role of individual Wnt proteins in osteogenesis needs to be investigated. The present study thus aimed to explore the role of Wnt7a in bone formation. For this purpose, human bone-derived MSCs were identified by flow cytometry and the cell differentiation potential, including osteogenic and adipogenic differentiation was examined. In order to explore the role of Wnt7a in MSC osteogenic differentiation, Wnt7a expression was measured at the mRNA and protein level following treatment with the osteogenic inducer, bone morphogenetic protein (BMP)4/7, and following the induction of osteogenic or adipogenic differentiation. The ectopic expression of Wnt7a in MSCs was confirmed and its influence on MSC osteogenic differentiation was detected using osteocyte markers and by Alizarin Red S staining. Mechanistically, the influence of Wnt7a on Runt-related transcription factor 2 (RUNX2) expression was examined at the mRNA and protein level. The regulatory effects of Wnt7a on RUNX2 promoter activities were examined by promoter reporter assay, and by examining
\end{abstract}

Correspondence to: Dr Xiaowei Dou, Clinical Research Center, The Affiliated Hospital of Guizhou Medical University, 28 Guiyi Street, Guiyang, Guizhou 550001, P.R. China

E-mail: daviddow@126.com

Dr Lixin Zhu, Department of Spinal Surgery, Orthopaedic Medical Center, Zhujiang Hospital, Southern Medical University, 253 Gongye Middle Avenue, Guangzhou, Guangdong 510282, P.R. China

E-mail: zhulixin1966@163.com

Key words: mesenchymal stem cell, osteogenesis, Wnt7a, RUNX2 the binding of TCF1, a downstream target of Wnt, to the RUNX2 promoter by ChIP assay. The results revealed that the knockdown of Wnt7a in MSCs decreased the expression of osteocyte markers and inhibited osteogenic differentiation. In accordance, the overexpression of Wnt7a in MSCs increased the expression of osteocyte markers and promoted osteogenic differentiation. Mechanistically, the knockdown of Wnt7a in MSCs reduced RUNX2 expression and the overexpression of Wnt7a in MSCs promoted RUNX2 expression. Furthermore, it was confirmed that Wnt7a regulated RUNX2 promoter activities by promoter report assay, and by examining the binding of TCF1 to the RUNX2 promoter by ChIP assay. On the whole, the present study demonstrates that Wnt7a plays a key role in MSC differentiation into osteoblasts and the findings presented herein may provide a promising therapy target for bone-related diseases.

\section{Introduction}

Bone marrow (BM)-derived mesenchymal stem cells (MSCs) are multipotent cells that have self-renewal capabilities and multilineage differentiation potential including bone, fat, cartilage and muscle (1). Understanding the process and molecular mechanisms of MSC differentiation to bone holds significant promise to provide new therapeutic strategy for bone-related diseases (2-5). To date, a number of clinical trials using MSCs for the treatment of bone-related diseases have been performed (6-9). A number of studies have focused on intrinsic transcription factors that regulate the differentiation of MSCs into osteocytes (10-12). However, their therapeutic utility still requires a more in-depth understanding of the molecular mechanisms that regulate MSC differentiation into osteoblasts.

There is evidence to suggest that Wnt signaling regulates osteoblast differentiation. The secreted glycoproteins, Wnts, and their receptors include at least $19 \mathrm{Wnts}, 10 \mathrm{Fzd}$ receptors, the two co-receptors, low-density lipoprotein receptor-related protein (LRP)5/LRP6 and several inhibitors, such as Dickkopf (Dkk)s, Frizzled (Fz)-related proteins (Frps) and Wif $(13,14)$. The canonical Wnt signaling is where Wnt binds 
to frizzled receptors and LRP-5 and/or LRP-6 co-receptors, promotes disheveled activation, and then blocks the function of glycogen synthase kinase (GSK)-3 $\beta$ (15). The inactivation of GSK-3 $\beta$ induces the cytoplasmic accumulation of $\beta$-catenin, which translocates to the nucleus and activates T-cell factor/lymphoid enhancer factor (TCF/LEF) family, leading to transcriptional activation of target genes (16). Previous research has indicated that the homozygous Wnt1 mutation gives rise to severe bone fragility and that the heterozygous Wnt1 mutation in family members tends to cause dominant early-onset osteoporosis; four children with homozygous or heterozygous Wnt1 mutations treated with bisphosphonate exhibited increased bone mineral density (17-20). In the study by Keller et al (21), the expression of Wnt5a significantly increased at day 7 and the expression of Wnt3a was observed at a later stage than that of Wnt5a during osteogenic induction. During mouse embryonic stem cell osteogenesis in vitro, the supplementation of Wnt5a from 5 to 7 days significantly enhanced osteogenic yield, although treatment with Wnt5a for the duration of the osteogenic induction period inhibited osteogenesis. Treatment with Wnt3a inhibited osteogenic differentiation from 5 to 7 days, but enhanced osteogenesis from 7 to 9 days. These intriguing results confirmed that Wnt5a and Wnt3a act sequentially in the osteogenic differentiation of mouse embryonic stem cell (21). A previous study using an engineered mouse model revealed that activation of Wnt7b dramatically enhanced bone mass (22). These results suggested the role of individual Wnts in osteogenic differentiation and bone formation; this warrants further investigations.

Of note, various individual Wnts have displayed distinct expression patterns in different bones. Wnt10b is expressed in all bones, whereas Wnt4 expression is higher in the trabecular endosteum, and Wnt7b is highly expressed in the perichondrium, indicating that there are various individual Wnt functions in different bones (23). In ST2 cells or 3T3-L1 cells, the overexpression of Wnt10a and Wnt10b has been shown to inhibit adipogenesis and promote osteogenesis; however, the depletion of Wnt6 increased adipocyte differentiation and reduced osteogenesis compared with the knockdown of Wnt10a or Wnt10b (24). These previous findings demonstrate that further research is required to focus on clarifying the roles of individual Wnts in osteogenic differentiation and bone formation.

Previously, msh homeobox 2 (Msx2) was found to be a regulator of osteogenic differentiation, and to upregulate Wnt7a expression; the knockdown of Wnt7a significantly reduced Msx2-induced alkaline phosphatase (25). To further explore the role of Wnt7a in the differentiation of MSCs into osteocytes, the present study examined the expression of Wnt7a in MSCs subjected to osteogenic- and adipogenic-induced differentiation. It was found that osteogenic induction medium increased Wnt7a expression, whereas adipogenic medium downregulated Wnt7a expression. The knockdown of Wnt7a in MSCs impaired osteogenic commitment in vitro and the enforced expression of Wnt7a in MSCs enhanced osteocyte formation in vitro. Mechanistically, it was found that Wnt7a significantly upregulated the expression of the osteogenic regulator, Runt-related transcription factor 2 (RUNX2).

\section{Materials and methods}

Ethics statement. Human bone tissues were obtained from following the orthopedic surgery of five 20- to 30-year-old male patients in 2016 at the Department of Orthopedics of the Affiliated Hospital of Guiyang Medical University. All experiments and protocols were approved by the Ethics Committee of Guizhou Medical University. Each patient provided written informed consent prior to the preparation of the bone tissues.

Cells and cell culture. Aseptic bone samples were minced with blades and then transferred to a digestion medium containing $200 \mathrm{U} / \mathrm{ml}$ collagenase I (Sigma-Aldrich; Merck KGaA) for $2 \mathrm{~h}$ at $37^{\circ} \mathrm{C}$ with intermittent agitation. The cell suspension was filtered through a $40-\mu \mathrm{m}$ strainer (BD Biosciences) and rinsed with D-Hanks solution twice. The cells were cultured in DMEM containing $10 \%$ fetal bovine serum (FBS), $100 \mathrm{U} / \mathrm{ml}$ penicillin and $100 \mu \mathrm{g} / \mathrm{ml}$ streptomycin. MSCs were used in the 6th passage. MSCs were treated with $150 \mathrm{ng} / \mathrm{ml}$ bone morphogenetic protein (BMP) $4 / 7$ (R\&D Systems) for $24 \mathrm{~h}$ and mRNA levels were determined by reverse transcription-quantitative PCR (RT-qPCR) at $24 \mathrm{~h}$ and protein levels by western blot analysis at $48 \mathrm{~h}$ as previously described (26).

Vector construction and viral infection. shRNA was purchased from Sigma-Aldrich; Merck KGaA and the target sequence for shWnt7a was as follows: 5'-CGTGCTCAAGGA CAAGTACAA-3'. Viral synthesis was performed according to manufacturer's instructions. Briefly, $2 \mu \mathrm{g}$ the shWnt7a or control vector were co-transfected with the packaging vectors, $2 \mu \mathrm{g}$ psPAX2 and $1 \mu \mathrm{g}$ pMD2G, into 293T cells (ATCC) in each well of a 6-well plate. For viral infection, cells were plated overnight and the MSCs were then infected in the presence of $8 \mu \mathrm{g} / \mathrm{ml}$ polybrene for $6 \mathrm{~h}$. The cells were selected with $2 \mu \mathrm{g} / \mathrm{ml}$ puromycin for 2 days. The sequence encoding human Wnt7a was amplified by RT-qPCR (primers: 5'-TTG GCGCGCCGCCACCATGAACCGGAAAGCGCGGCG-3' and 5'-CCTTAATTAATCACTTGCACGTGTACATCT-3') from pcDNA-Wnt7A-V5 (Addgene) (27) and inserted into the AscI/PacI sites of pCDF1-MCS2-EF1-copGFP (SBI). pCDF1-Wnt7a or pCDF1 were co-transfected with packaging vector pFIV34N and pVSV-G overnight. At $48 \mathrm{~h}$ following transfection, the virus was collected and used to infect the MSCs in the presence of $8 \mu \mathrm{g} / \mathrm{ml}$ polybrene for $6 \mathrm{~h}$. The Wnt7a-positive cells were isolated by FACS analysis using GFP. Briefly, $1 \times 10^{6} \mathrm{pCDF} 1-W n t 7 a$ or pC DF1 infected MSCs were digested by $0.05 \%$ trypsin $/ 0.02 \%$.

EDTA and filtered with a $40 \mu \mathrm{m}$ strainer. The cells were rinsed 3 times by D-Hanks and sorted using a BD FACSAria III cell sorter. The sorted cells were cultured in a $6-\mathrm{cm}$ culture dish (Corning, Inc.).

Immunofluorescence staining. MSCs were fixed in 4\% PFA at room temperature for $15 \mathrm{~min}$ and permeabilized with $0.5 \%$ Triton X-100 for $5 \mathrm{~min}$. The cells were blocked with $2.5 \%$ goat serum for $1 \mathrm{~h}$ and then stained with 1:200 anti-Ki-67 mouse monoclonal antibody (M7240; Dako; Agilent Technologies, Inc.) overnight at $4^{\circ} \mathrm{C}$. Antibody binding was visualized by incubation with a FITC-conjugated goat anti-mouse secondary antibody (1:200, sc-2010; Santa Cruz Biotechnology, Inc.) at 
room temperature for $1 \mathrm{~h}$. Mouse IgG2a (1:200, sc-2856; Santa Cruz Biotechnology, Inc.) was used as the isotype control for Ki-67 antibody and FITC-conjugated goat anti-mouse antibody (1:200, sc-2010; Santa Cruz Biotechnology, Inc.) as a negative control to assess non-specific binding of the secondary antibody. MSCs were then stained with DAPI at room temperature for $3 \mathrm{~min}$ and imaged using an Olympus BX51 fluorescence microscope.

Flow cytometry. MSCs were digested with $0.05 \%$ trypsin/ $0.02 \%$ EDTA and filtered with a $70-\mu \mathrm{m}$ strainer. The cells were blocked with $2.5 \%$ goat serum for $30 \mathrm{~min}$ and stained with anti-CD44 (555478), -CD90 (561969), -CD105 (561443), -CD34 (555821) and -CD45 (555482) antibodies at a dilution of 1:200 for $45 \mathrm{~min}$. Rat IgG2a was used as an isotype control. All antibodies were directly conjugated to FITC and obtained from BD Biosciences. MSCs were rinsed 5 times and analyzed on an Epics XL flow cytometer (Beckman Coulter, Inc.).

Alizarin Red S staining. To induce osteogenic differentiation, MSCs were grown in DMEM supplemented with $10 \%$ FCS, $10 \mathrm{mM} \beta$-glycerophosphate, $10^{-7} \mathrm{M}$ dexamethasone and $0.2 \mathrm{mM}$ ascorbic acid (all from Sigma-Aldrich; Merck KGaA). The medium was changed every three days. Following two weeks of culture in vitro, the cells were fixed in $10 \%$ formalin for $15 \mathrm{~min}$ at room temperature and stained by Alizarin Red S stain solution for $30 \mathrm{~min}$ at room temperature using the Osteogenesis Assay kit according to the manufacturer's instructions (EMD Millipore).

Oil Red $O$ staining. To induce adipogenic differentiation, MSCs were grown in DMEM containing $0.5 \mu \mathrm{M}$ hydrocortisone, $0.5 \mathrm{mM}$ isobutylmethylxanthine and $50 \mu \mathrm{g} / \mathrm{ml}$ indomethacin (all from Sigma-Aldrich; Merck KGaA) (28). The medium was changed every three days. Following two weeks of culture, the cells were fixed with $10 \%$ formalin and stained with fresh Oil Red O solution for $10 \mathrm{~min}$ at room temperature (Sigma-Aldrich; Merck KGaA) to detect lipid droplets.

$R T-q P C R$. TRIzol $^{\circledR}$ reagent (Invitrogen; Thermo Fisher Scientific, Inc.) was used to isolate total RNA from the MSCs. cDNA was synthesized using the PrimeScript ${ }^{\mathrm{TM}}$ RT Reagent kit (Takara Bio, Inc.) according to the manufacturer's protocol. qPCR was performed using SYBR Premix Ex Taq II (Takara Bio, Inc.) and the CFX96 Real-time PCR Detection system (Bio-Rad Laboratories, Inc.). Each sample is done in triplicate. The primers used were as follows: $\beta$-actin forward, 5'-gtaccactggcatcgtgatggact-3' and reverse, 5'-ccgctcattgccaatggtgat-3'; osteocalcin (OCN) forward, 5'-AGCAAAGGT GCAGCCTTTGT -3' and reverse, 5'-GCGCCTGGGTCT CTTCACT-3'; osteopontin (OPN) forward, 5'-ATGATGGCC GAGGTGATAGT-3' and reverse, 5'-ACCATTCAACTCCTC GCTTT-3'; peroxisome proliferator-activated receptor G2 (PPARG2) forward, 5'-TCCATGCTGTTATGGGTGAA-3' and reverse, 5'-TCAAAGGAGTGGGAGTGGTC-3'; lipoprotein lipase $(\mathrm{Lpl})$ forward, 5'-AGTGGCCAAATAGCACAT CC-3' and reverse, 5'-CCGAAAGATCCAGAATTCCA-3'; Wnt7a forward, 5'-CTGTGGCTGCGACAAAGAGAA-3' and reverse, 5'-GCCGTGGCACTTACATTCC-3'; RUNX2 forward, 5'-CAGTAGATGGACCTCGGGAA-3' and reverse,
5'-CCTAAATCACTGAGGCGGTC-3'; and osterix (OSX) forward, 5'-AGCCTCAGGATGGCGTC-3' and reverse, 5'-AGAGTTGTTGAGTCCCGCAG-3'. The results were analyzed using the $2^{-\Delta \Delta \mathrm{Cq}}$ method (29).

Western blot analysis. To obtain protein extracts, MSCs were washed with chilled PBS and lysed with RIPA buffer containing a protease inhibitor cocktail (Roche Diagnostics). The protein concentration was measured using BCA methods according to the manufacturer's instruction (P0012S, Beyotime) and $30 \mu \mathrm{g}$ were separated by $10 \%$ SDS-PAGE gel and transferred to PVDF membranes. The protein was blocked by $5 \%$ milk for $1 \mathrm{~h}$ at room temperature. The membranes were incubated with specific primary antibodies against $\beta$-actin (sc-47778, Santa Cruz Biotechnology, Inc.), Wnt7a (ab100792; Abcam), OCN (ab13418; Abcam), OPN (ab214050; Abcam), RUNX2 (12556, Cell Signaling Technology, Inc.) and OSX (ab94744, Abcam) at 1:1,000 overnight at $4^{\circ} \mathrm{C}$. The membranes were rinsed three times by TBST and incubated with 1:1,000 goat against rabbit (sc-2357; Santa Cruz Biotechnology, Inc.) or 1:1,000 m-IgGкBP-HRP (sc-516102; Santa Cruz Biotechnology, Inc.) horseradish peroxidase-conjugated antibodies for $1 \mathrm{~h}$ at room temperature. The bands were detected by Immobilon Western Chemiluminescent HRP Substrate (ECL, WBKLS0100; Thermo Fisher Scientific, Inc.).

Luciferase assays. The TCF1-binding region (-900 to -1,200 bp upstream of RUNX2 exon 1) was cloned by PCR amplification from 293T genomic DNA and inserted into the $\mathrm{SacI} / \mathrm{XhoI}$ sites of the pGL3-control vector (Promega Corporation). The primers for cloning the TCF1-binding region in RUNX2 promoter were as follows: Forward, 5'-GCGCGAGCTCAGGGGCAAAAA AGGAGATAGTT-3'; and reverse, 5'-GCGCCTCGAGGA GTTTCTGATAGCAGATCTTCTAT-3'. pRL-SV40 (Promega Corporation) was co-transfected as an internal control to assess the transfection efficiency. Wnt7a silencing/control or Wnt7a overexpressing/control MSCs were grown in osteogenic-inducing medium for 7 days. A total of $100 \mu \mathrm{g}$ the reporter constructs were co-transfected with $10 \mathrm{ng}$ pRL-SV40 into MSCs in each well of 96-well plate in which Wnt7a was silenced or overexpressed with internal control plasmids. Cells were collected following $24 \mathrm{~h}$ of transfection, and luciferase activities were detected using the Dual-Luciferase Reporter assay (Promega Corporation) according to the manufacturer's protocol. The relative promoter activities were expressed as the fold change in Firefly luciferase activity following normalization to Renilla luciferase activity. A total of three independent experiments were performed for each sample.

ChIP assays. The assays were performed using a ChIP assay kit according to the manufacturer's protocol (P2078; Beyotime Institute of Biotechnology). The antibodies used for CHIP assays were purchased from the following companies: TCF1 (2203, Cell Signaling Technology, Inc.) and IgG (sc-2003; Santa Cruz Biotechnology, Inc. as an IP control). A total of $2 \mu \mathrm{g}$ TCF1 or IgG were incubated with the cell lysates overnight at $4^{\circ} \mathrm{C}$ as previously reported (30). As previously reported, TCF1 recognizes and binds to the consensus motif TTCAAAG $(31,32)$. PCR generated a product from the RUNX2 promoter containing a TCF1-binding site $(-1,056$ to $-1,062 \mathrm{bp}$ 
A

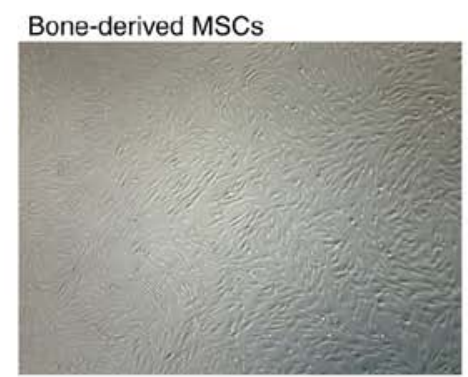

$B$

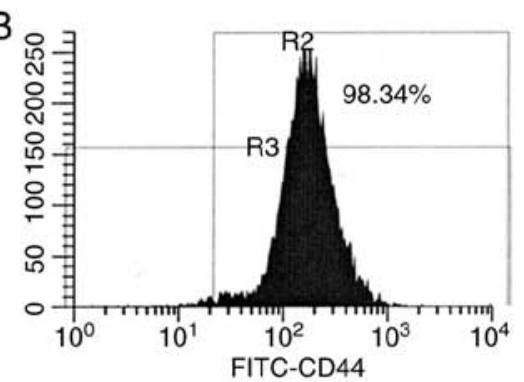

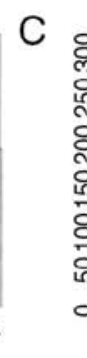

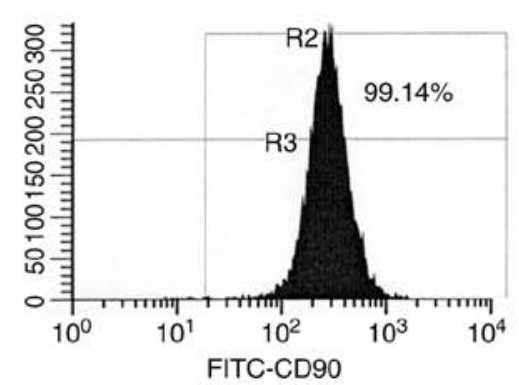

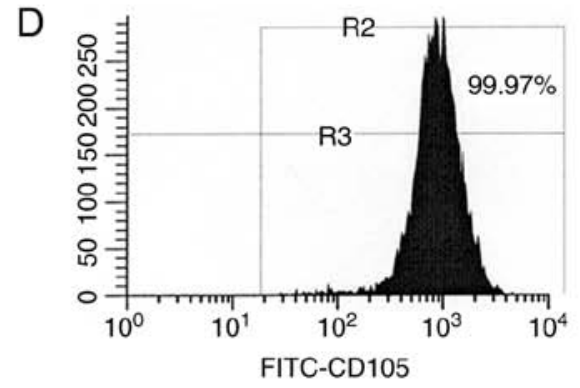
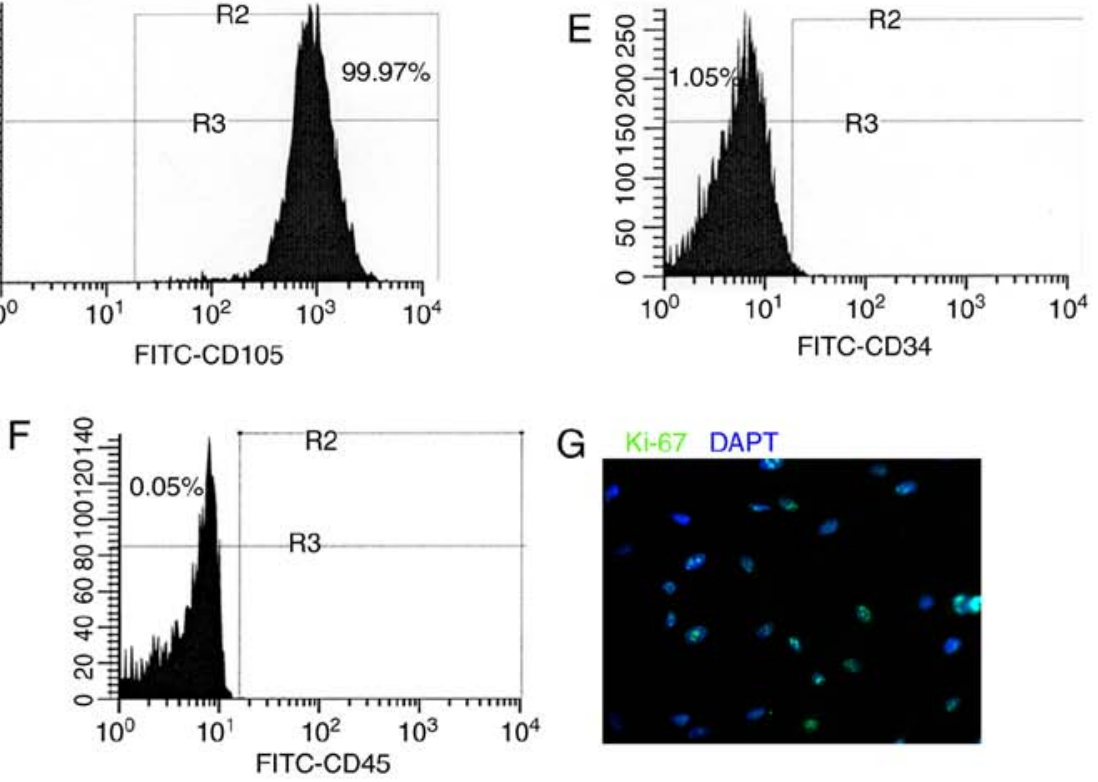

Figure 1. Characterization of human MSCs. (A) MSCs displayed a fibroblast-like morphology at the sixth passage. (B-D) FACS analysis revealed positive staining for CD44, CD90 and CD105, respectively in MSCs. (E and F) FACS analysis revealed negative staining for the hematopoietic lineage markers, CD34 and CD45, respectively in MSCs. (G) Ki-67 staining revealed the proliferative ability of MSCs. MSCs, mesenchymal stem cells.

upstream of exon 1) or a product from the distal region $8 \mathrm{~kb}$ downstream for RUNX2 without the TCF1-binding site. Each experiment was performed in three samples. The primers used for RT-qPCR for the TCF1-binding element in the RUNX2 promoter were as follows: Forward, 5'-CTGAATCAGAAT TAGCAAATCG-3' and reverse, 5'-GTTTCTAATGGAAGC TTTGA-3'; and $8 \mathrm{~kb}$ downstream for RUNX2 forward, 5'-GAT TGCAAAAAACAGATATTAA-3' and reverse, 5'-GAAAGT CTTGTGTGACTAAATA-3'.

Statistical analysis. All experiments were performed in triplicate. The variables were compared between groups by a paired samples t-test. $\mathrm{P}<0.05$ was considered to indicate a statistically significant difference. Statistical analysis was carried out using SSPS 13.0 software.

\section{Results}

Phenotype of cultured BM-derived MSCs. The BM-derived cells were cultured in DMEM and the phenotype was detected in the sixth passage. The adherent BM-derived cells exhibited a fibroblast-like morphology (Fig. 1A). Flow cytometry was performed to characterize the phenotype of the BM-derived cells used in the present study. These BM-derived cells expressed the MSC markers, CD44,
CD90 and CD105 (Fig. 1B-D), whereas they did not express the hematopoietic lineage markers, CD34 and CD45 (Fig. 1E and F). Immunofluorescence staining revealed that the BM-derived cells comprised more Ki-67-labeled proliferative cells (Fig. 1G). These findings suggested that the BM-derived cells used in the present study acquired the phenotype and characteristics of MSCs.

In vitro differentiation potential of MSCs. To evaluate the differentiation potential of the MSCs used in the present study, osteogenic medium and adipocyte-specific induction medium were used to culture the MSCs. Following two weeks of induction with osteogenic medium, it was demonstrated that the MSCs cultured with osteogenic medium exhibited osteogenic differentiation capacity compared with the MSCs without osteogenic induction, as evidenced by mineralized nodule formation assessed by Alizarin Red S staining (Fig. 2A). The results of RT-qPCR confirmed that the MSCs cultured with osteogenic medium for 6 days expressed higher levels of the osteogenic markers, OCN and OPN (Fig. 2B and C). The MSCs also exhibited adipogenic differentiation capacity, as confirmed by Oil Red $\mathrm{O}$ staining of adipocytes induced by growing MSCs in adipocyte-specific induction medium for two weeks (Fig. 2D). The results of RT-qPCR revealed that the expression levels of the adipogenic markers, PPARG2 and Lpl 
A

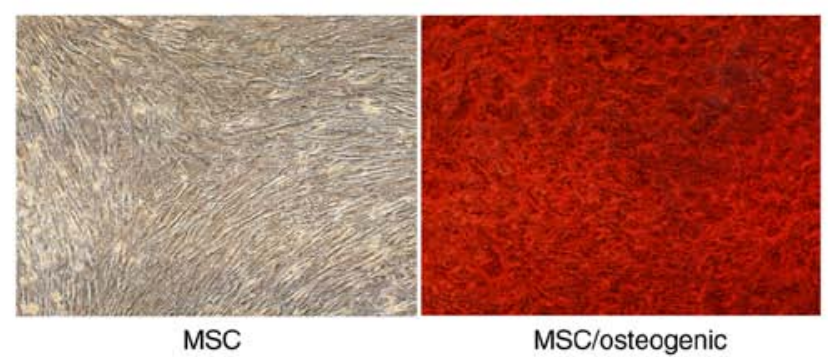

MSC

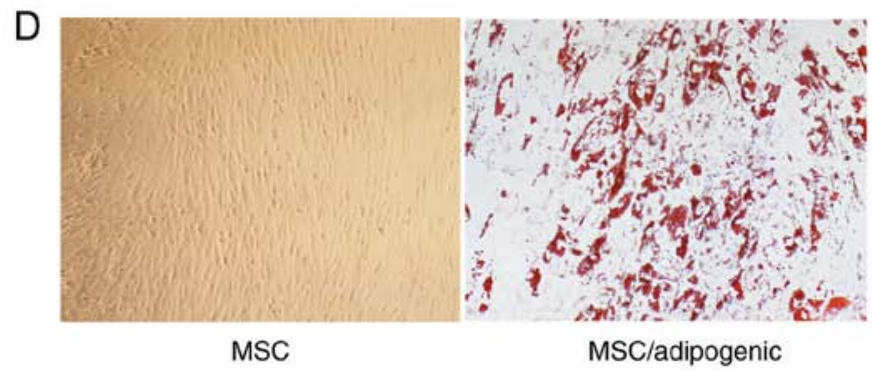

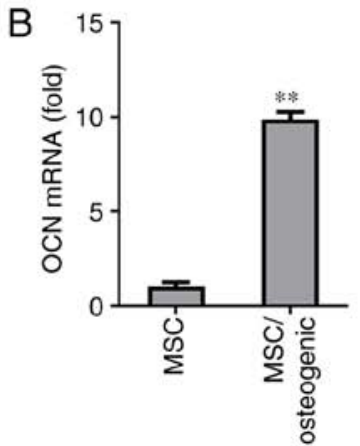
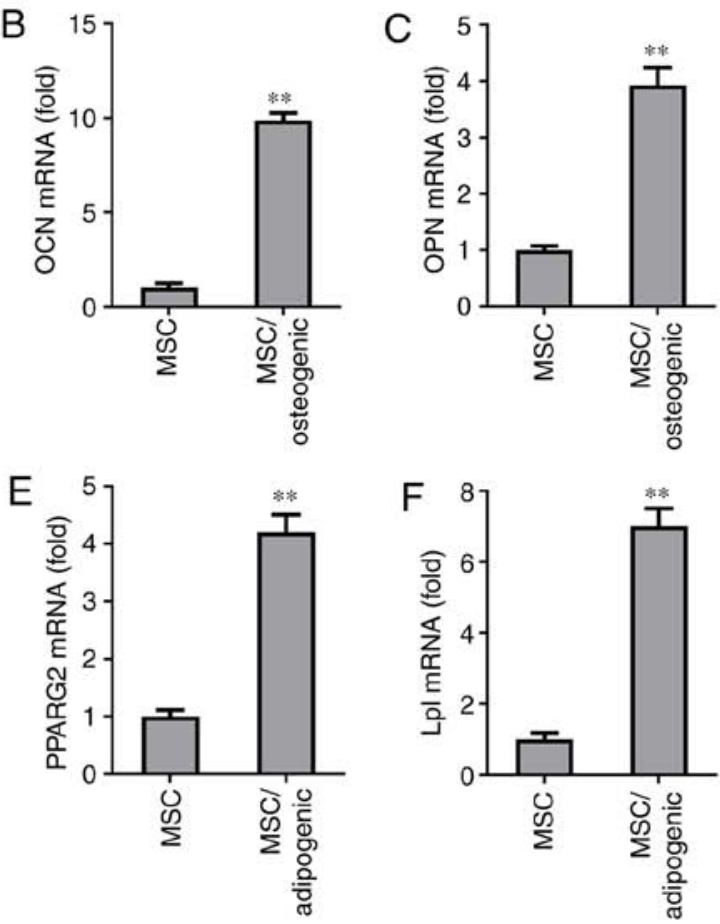

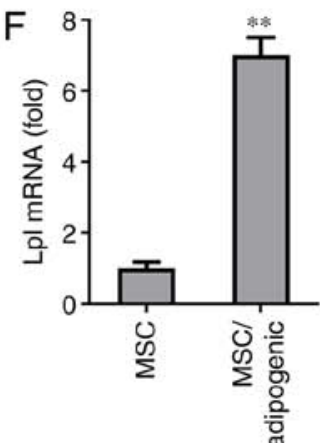

Figure 2. The differentiation potential of MSCs. (A) Alizarin Red S staining revealed the osteogenic differentiation of MSCs. (B and C) RT-qPCR confirmed the higher expression of the osteogenic markers, OCN and OPN, in osteogenically differentiated MSCs. ${ }^{* *} \mathrm{P}<0.01$ vs. MSCs without osteogenic differentiation. (D) Oil Red O staining displayed the adipogenic differentiation of MSCs. (E and F) RT-qPCR revealed the elevated expression of the adipogenic markers, PPARG2 and Lpl in adipogenically differentiated MSCs. ${ }^{* *} \mathrm{P}<0.01$ vs. MSCs without adipogenic differentiation. MSCs, mesenchymal stem cells; OCN, osteocalcin; OPN, osteopontin; PPARG2, peroxisome proliferator-activated receptor gamma isoform-2; Lpl, lipoprotein lipase.

were higher after the MSCs were grown in adipocyte-specific induction medium for six days (Fig. 2E and F). These findings indicated that the MSCs used in the present study acquired multilineage differentiation potential.

Induction of Wnt7a expression by the osteogenic differentiation of MSCs. Since BMP-4/7 are capable of inducing MSC differentiation into osteocytes, the present investigated whether the expression of Wnt7a was induced by BMP-4/7 in MSCs. The results of RT-qPCR revealed that Wnt7a expression was strongly induced by BMP4/7 (Fig. 3A). Western blot analysis revealed that the expression of Wnt7a was elevated at the protein level in BMP4/7-treated MSCs (Fig. 3B). The expression of Wnt7a was also examined in MSCs cultured in osteogenic-inducing medium. Notably, after six days of culture, the expression of Wnt7a was found to be elevated in MSCs cultured in osteogenic-inducing medium compared with the MSCs without osteogenic-inducing medium, as shown by RT-qPCR and western blot analysis (Fig. 3C and D). Since the dynamic balance between osteogenesis and adipogenesis exists in MSC differentiation, the regulators that promote an osteogenic lineage commitment could be inhibited under the condition of adipogenic differentiation. To explore whether the expression of Wnt7a was regulated during the adipogenic differentiation of MSCs, Wnt7a expression was determined at the mRNA and protein level in the MSCs grown in adipogenic-inducing medium for six days. The results of RT-qPCR revealed that Wnt7a expression was significantly inhibited in MSCs grown in adipogenic-inducing medium (Fig. 3E). Consistently, western blot analysis revealed that the expression of Wnt7a was inhibited in MSCs grown in adipogenic-inducing medium (Fig. 3F). Since the Wnt signal pathway plays a central role in the osteogenic differentiation of MSCs, these findings suggested that Wnt7a plays a critical role in MSC differentiation into osteocytes.

Knockdown of Wnt7a reduces the osteogenic differentiation of MSCs. To directly determine whether Wnt7a plays a critical role in the osteogenic differentiation of MSCs, lentivirus was used to stably silence its expression in MSCs. The knockdown of Wnt7a in MSCs was confirmed by RT-qPCR (Fig. 4A). Consistently, western blot analysis revealed that lentivirus expressing Wnt7a shRNA reduced Wnt7a expression at the protein level (Fig. 4B). Following osteogenic induction for 6 days, RT-qPCR revealed that the knockdown of Wnt7a significantly reduced the expression of OCN, an early marker of osteogenic differentiation (Fig. 4C). Consistently, western blot analysis revealed that knockdown of Wnt7a reduced the expression of OCN at the protein level (Fig. 4D). Similarly, the knockdown of Wnt7a reduced the mRNA and protein expression of OPN, another early marker of osteogenic differentiation (Fig. 4E and F). Furthermore, the results revealed that the knockdown of Wnt7a in MSCs reduced the formation of mineralized nodules after two weeks of culture in osteogenic-inducing medium (Fig. 4G). These findings implied that the suppression of Wnt7a expression inhibited the osteogenic differentiation of MSCs in vitro.

Overexpression of Wnt7a promotes osteogenic differentiation. To further examine whether Wnt7a plays a critical role in the osteogenic differentiation of MSCs, Wnt7a was overexpressed in MSCs using lentivirus. The overexpression of 

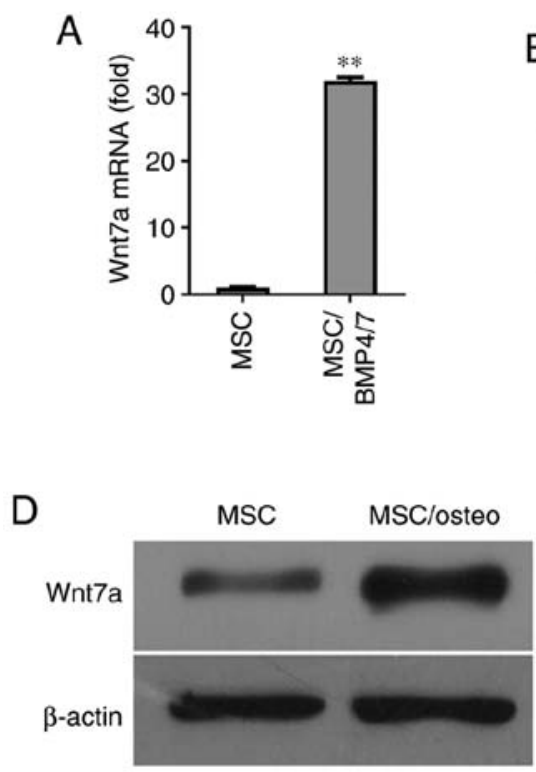
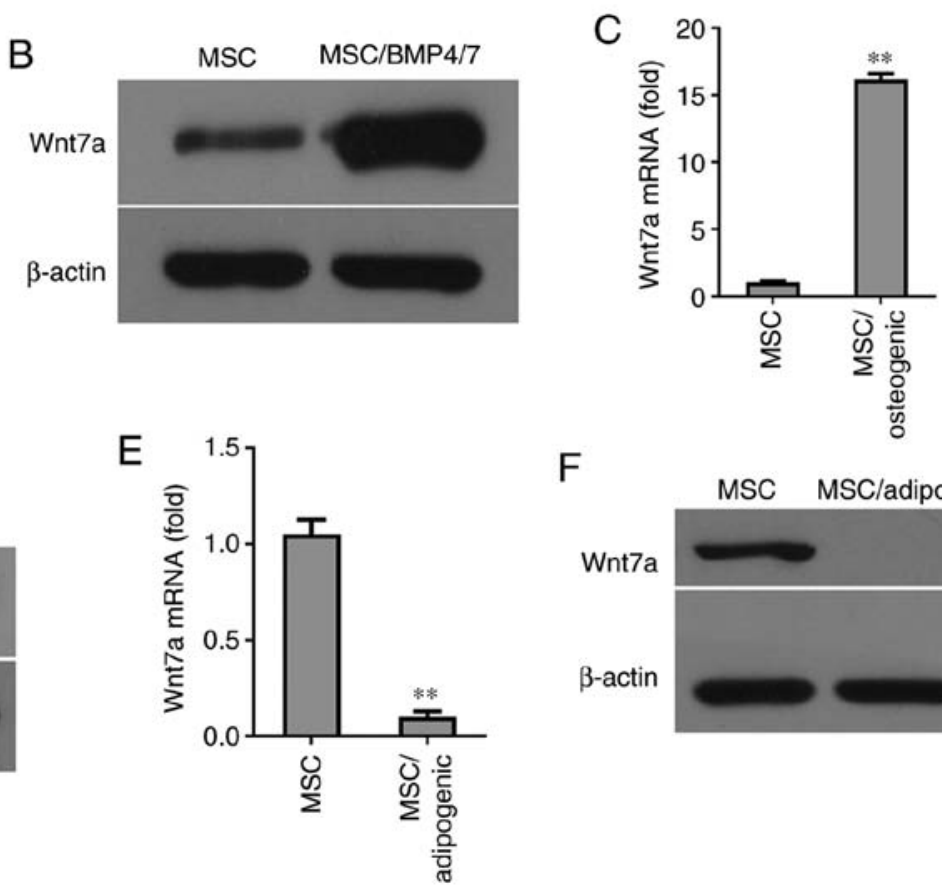

$\mathrm{F}$

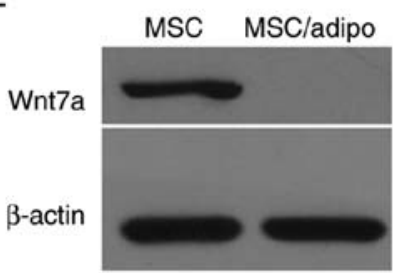

Figure 3. Induction of Wnt7a expression by osteogenesis. (A and B) RT-qPCR and western blot analysis revealed the elevated expression of Wnt7a in BMP4/7-treated MSCs. ${ }^{* *} \mathrm{P}<0.01$ vs. untreated MSCs. (C and D) RT-qPCR and western blot analysis revealed the higher expression of Wnt7a in osteogenically differentiated MSCs. ${ }^{* *} \mathrm{P}<0.01$ vs. MSCs without osteogenic differentiation. (E and F) RT-qPCR and western blot analysis revealed the reduced expression of Wnt7a in adipogenically differentiated MSCs. ${ }^{* *} \mathrm{P}<0.01$ vs. MSCs without adipogenic differentiation. MSCs, mesenchymal stem cells; BMP, bone morphogenetic protein.

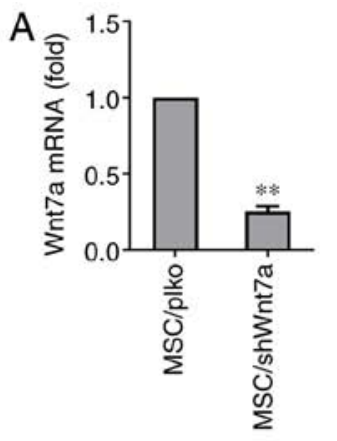

B
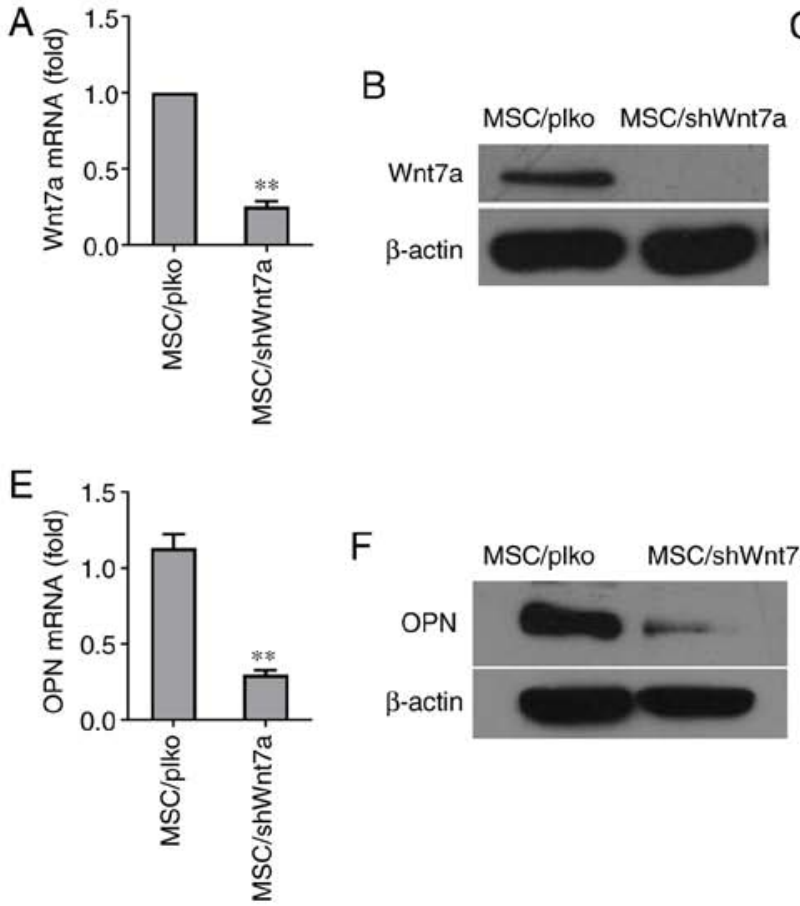
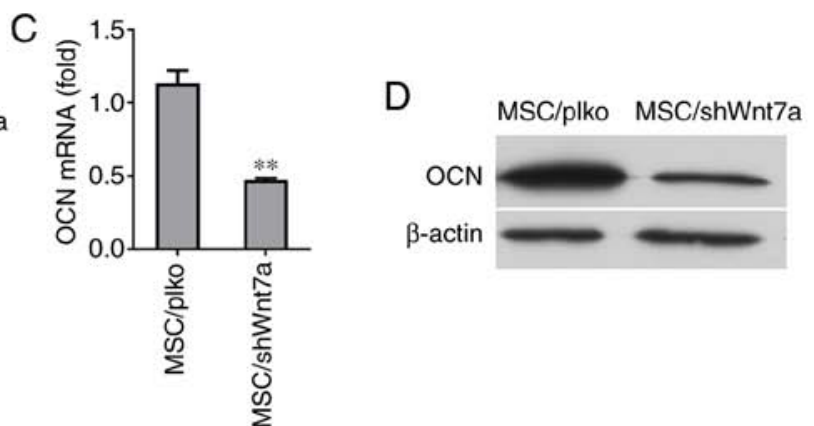

G

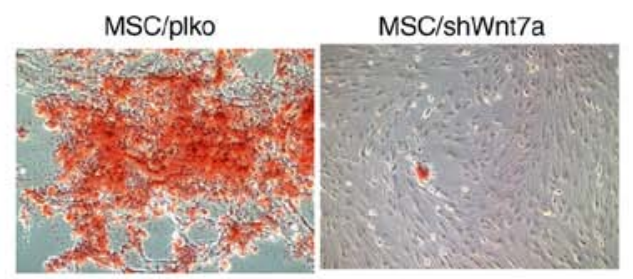

Figure 4. Suppression of osteogenesis by depletion of Wnt7a. (A and B) Knockdown of Wnt7a by shRNA. Expression of Wnt7a was detected by RT-qPCR and western blot analysis. ${ }^{* *} \mathrm{P}<0.01$ vs. control. (C and D) Knockdown of Wnt7a inhibited OCN expression. OCN expression was determined by RT-qPCR and western blot analysis. ${ }^{* *} \mathrm{P}<0.01$ vs. control. (E and F) Depletion of Wnt7a suppressed OPN expression. OPN expression was determined by RT-qPCR and western blot analysis. ${ }^{* *} \mathrm{P}<0.01$ vs. control. (G) Depletion of Wnt7a suppressed mineralized nodule formation. Mineralized nodules were determined by Alizarin Red $\mathrm{S}$ staining. MSCs, mesenchymal stem cells; OCN, osteocalcin; OPN, osteopontin.

Wnt7a in MSCs was confirmed at the mRNA level and protein level by RT-qPCR and western blot analysis, respectively (Fig. 5A and B). Following growth in osteogenic-inducing medium for six days, the Wnt7a-overexpressing MSCs exhibited a enhanced expression of OCN and ONN at the mRNA level and protein level (Fig. 5C-F). Moreover, it was found that the overexpression of Wnt7a in MSCs increased mineralization in vitro for 9 days, based on Alizarin Red S 

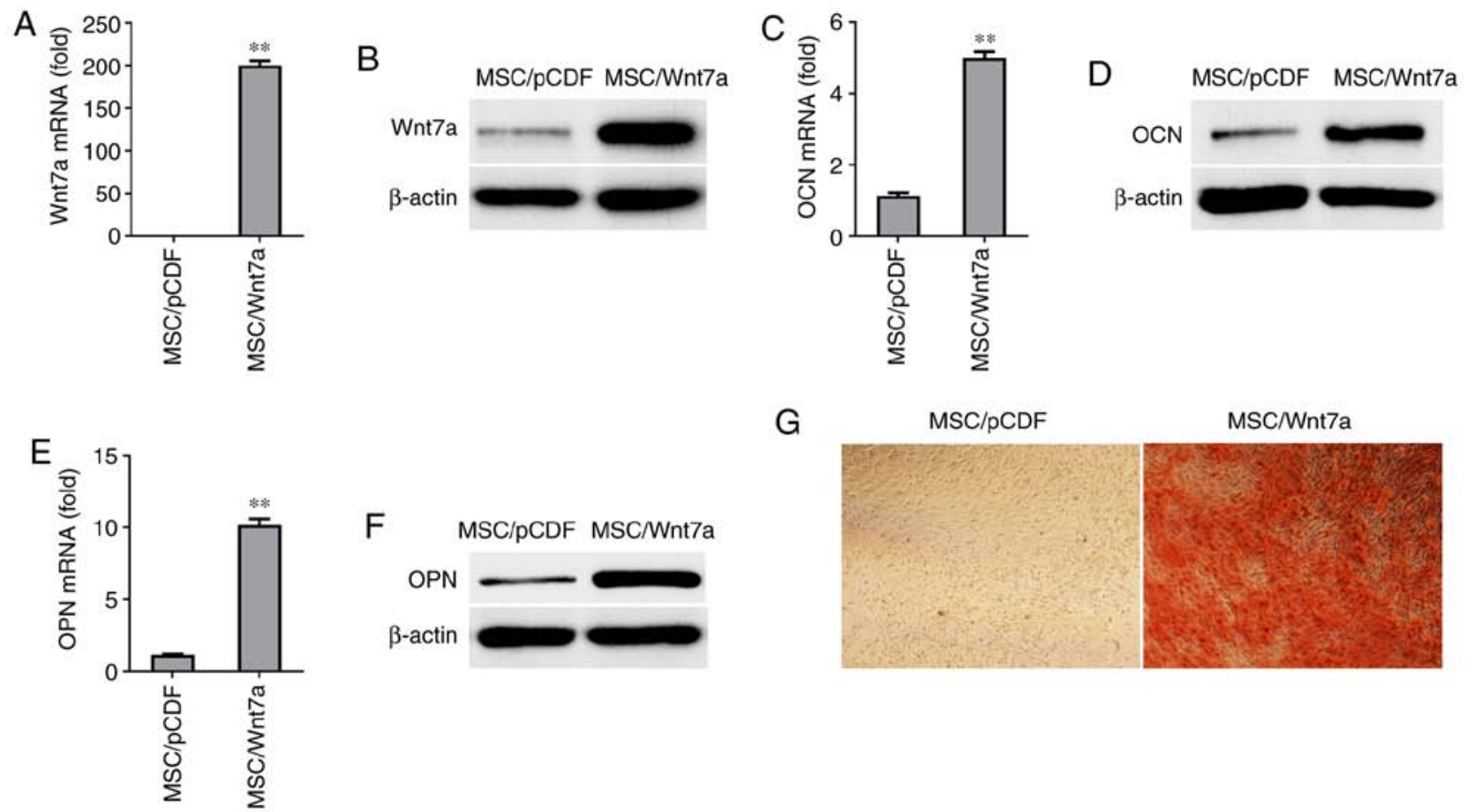

G

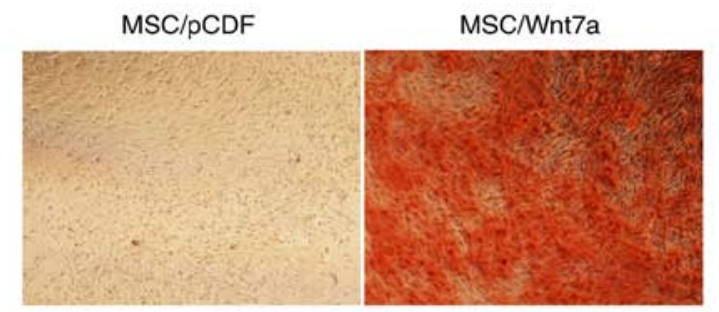

Figure 5. Promotion of osteogenesis by the enforced expression of Wnt7a. (A and B) The enforced expression of Wnt7a. Wnt7a expression was determined by RT-qPCR and western blot analysis, respectively. ${ }^{* *} \mathrm{P}<0.01$ vs. control. (C and D) Overexpression of Wnt7a increased OCN expression. OCN expression was determined by RT-qPCR and western blot analysis. ${ }^{* *} \mathrm{P}<0.01$ vs. control. (E and F) Overexpression of Wnt $7 \mathrm{a}$ increased OPN expression. OPN expression was determined by RT-qPCR and western blot analysis. ${ }^{* *} \mathrm{P}<0.01$ vs. control. (G) Overexpression of Wnt7a promoted mineralized nodule formation. Mineralized nodules were determined by Alizarin Red S staining. MSCs, mesenchymal stem cells; OCN, osteocalcin; OPN, osteopontin.

staining (Fig. 5G). These findings suggested that overexpression of Wnt7a enhanced the osteogenic differentiation of MSCs in vitro.

Wnt7a regulates osteogenic differentiation through RUNX2. To further explore the mechanisms underlying the regulation of osteogenic differentiation by Wnt7a, the present study examined the effects of Wnt7a on the expression of RUNX2 and OSX, which were previously confirmed to be the key regulators of osteogenic differentiation (33-36). Notably, following the growth of MSCs in osteogenic-inducing medium for six days, it was found that the knockdown of Wnt7a decreased the expression of RUNX2 at the mRNA level and protein level (Fig. 6A and B). However, RT-qPCR and western blot analysis revealed that the knockdown of Wnt7a in MSCs did not affect OSX expression at the mRNA level and protein level (Fig. 6A and B). The effects of Wnt7a overexpression on the expression of RUNX2 and OSX in MSCs were furthermore determined. Following the growth of MSCs in osteogenic-inducing medium for six days, RT-qPCR and western blot analysis revealed that the overexpression of $\mathrm{Wnt} 7 \mathrm{a}$ in MSCs increased RUNX2 expression at the mRNA level and protein level, but did not affect OSX expression at the mRNA level and protein level (Fig. 6C and D). To determine whether Wnt7a regulates MSC differentiation through RUNX2, the putative TCF1-binding site in the promoter of the RUNX2 gene was identified and a reporter construct was generated. Transient transfection assays demonstrated that the knockdown of Wnt7a in MSCs decreased RUNX2 promoter activity and that Wnt7a overexpression increased RUNX2 promoter activity (Fig. 6E-G). CHIP assays were further performed to assess the binding of TCF1-binding site to the promoter of RUNX2. Indeed, RT-qPCR revealed that the knockdown of Wnt7a in MSCs reduced TCF-1 binding to the RUNX2 regulatory region and that the overexpression of Wnt7a increased TCF1 binding to the RUNX2 regulatory region, indicating that Wnt7a regulates RUNX2 expression by TCF1 binding to the RUNX2 promoter (Fig. $6 \mathrm{H}-\mathrm{J}$ ). These findings suggest that Wnt7a regulates osteogenic differentiation through the key osteogenic regulator RUNX2.

\section{Discussion}

MSCs acquire multiple differentiation potential, including the capacity to differentiate into osteocytes or adipocytes $(1,37-39)$. The associated regulators include the Wnt/ $\beta$-catenin, transforming growth factor (TGF) $\beta / \mathrm{BMP}$, fibroblast growth factor (FGF), Notch and Hedgehog signaling pathways, and RUNX2, OSX, activating transcription factor 4 (ATF4), Tafazzin (TAZ) and nuclear factor of activated T-cells (NFATc) 1 transcriptional factors have been identified as being involved in MSC differentiation into osteocytes (40-43). Although there has been significant progress made in understanding the molecular framework in which MSCs differentiate into osteocytes, the underlying mechanisms controlling this process remain unclear. In the present study, it was demonstrated that Wnt7a played a critical role in MSC commitment to bone formation. Wnt7a was found to be upregulated during the osteogenic differentiation of MSCs but downregulated following MSC adipogenic differentiation. It was further confirmed that the knockdown of Wnt7a in MSCs suppressed the expression of osteocyte molecular markers and inhibited osteogenesis 

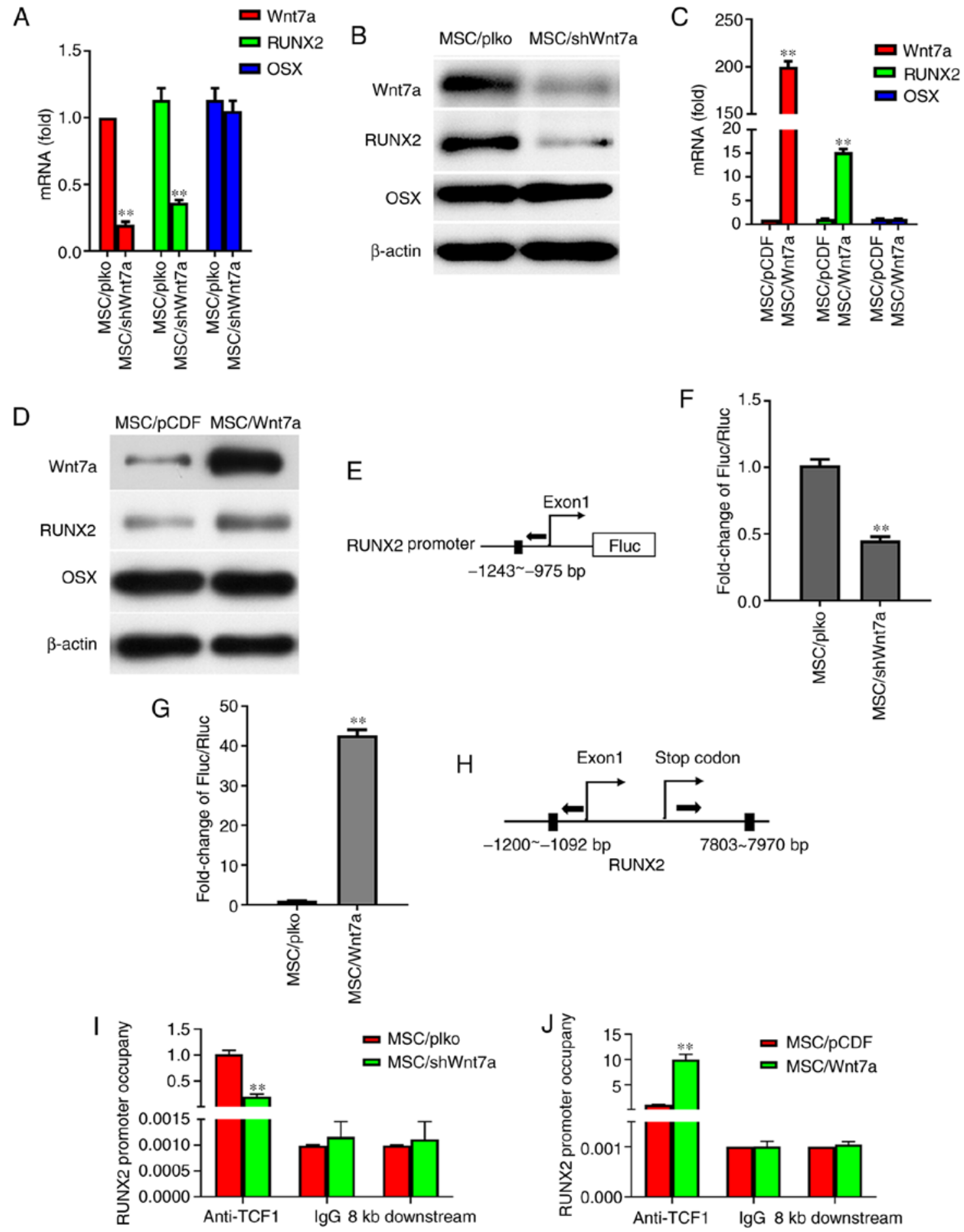

Figure 6. Wnt7a regulates osteogenesis through RUNX2. (A and B) Depletion of Wnt7a downregulated RUNX2 expression but did not affect OSX expression. RUNX2 and OSX expression were determined by RT-qPCR and western blot analysis. ${ }^{* *} \mathrm{P}<0.01$ vs. control. (C and D) Overexpression of Wnt7a increased RUNX2 expression but did not affect OSX expression. The expression of RUNX2 and OSX were determined by RT-qPCR and western blot analysis. ${ }^{* *} \mathrm{P}<0.01$ vs. control. (E) Schematic representation of the RUNX2 promoter region containing the TCF1-binding site and the reporter construct. (F) Knockdown of Wnt7a decreased RUNX2 promoter activities. Co-transfection of RUNX2 promoter construct and pRL-SV40 with shWnt7a or control vector in MSCs grown in osteogenic-inducing medium for 6 days. ${ }^{* *} \mathrm{P}<0.01$ vs. control. (G) Overexpression of Wnt7a enhanced RUNX2 promoter activities. Co-transfection of RUNX2 promoter construct and pRL-SV40 with Wnt7a or NT in MSCs grown in osteogenic-induction medium for 6 days. ${ }^{* *} \mathrm{P}<0.01$ vs. control. (H) Schematic representation of the PCR products of the TCF1 binding site or $8 \mathrm{~kb}$ downstream. (I) CHIP assay analysis. RT-qPCR revealed that the knockdown of Wnt7a decreased TCF1 occupancy on the RUNX2 promoter. ${ }^{* *} \mathrm{P}<0.01$ vs. control. (J) ChIP assay. RT-qPCR revaeled that the enforced expression of Wnt7a promoted TCF1 occupancy on the RUNX2 promoter. ${ }^{* *} \mathrm{P}<0.01$ vs. control. MSCs, mesenchymal stem cells; RUNX2, Runt-related transcription factor 2; OSX, osterix.

in vitro. Conversely, the overexpression of Wnt7a in MSCs promoted osteocytes markers expression and osteogenesis in vitro. The finding that Wnt7a regulates bone formation provides the opportunity to understand the pathogenic causes of bone-related diseases and to develop target therapies for these diseases.

Increasing evidence has demonstrated that Wnt signaling plays an important role in regulating MSC osteogenic 
differentiation. This concept is supported by the fact that Wnt1, Wnt3a and Wnt10b stimulate bone formation by activating $\beta$-catenin, while Dkk1, which suppresses the Wnt pathway, reduces osteocyte differentiation. The demonstration that Msx2 increased osteogenic differentiation and that the depletion of Msx2 reduced Wnt7a mRNA levels implied that Wnt7a may be implicated in the regulation of osteogenic differentiation (25). The results confirmed that the downregulation of Wnt7a expression decreased bone formation in vitro and that the enforced expression of Wnt7a promoted osteogenesis in vitro, suggesting that Wnt7a plays a key regulator role in osteocyte development. RUNX2 and OSX are essential regulators of osteogenic differentiation. Nakashima et al (44) demonstrated that OSX acted genetically downstream of RUNX2 in mesenchymal differentiation into osteocytes. Artigas et al (45) found that p53 inhibited OSX expression in osteoblast differentiation, but did not affect RUNX2 expression. The present study demonstrated Wnt7a regulated RUNX2 expression but did not affect the OSX level following osteogenic differentiation. Upon Wnt interaction with the receptors, FZD and LRP5/6, $\beta$-catenin accumulates in the nucleus, which releases histone deacetylases (HDACs) from the TCF and recruits histone acetylase CBP/p300 to activate downstream gene expression $(46,47)$. Previous research has indicated that Wnt signaling promotes osteogenic differentiation and bone-related gene expression through TCF binding to the consensus motif A/TA/TCAAAG of the promoters of these genes $(32,48)$. The present study also found that Wnt7a enhanced TCF-1 binding to the consensus motif TTCAAAG of the RUNX2 promoter. These results support the findings that Wnt7a promotes MSC differentiation into osteocytes possibly by regulating RUNX2 expression.

In conclusion, the findings of the present study demonstrated the role of Wnt7a in the differentiation of MSCs into osteocytes in vitro. Since Wnt7a is secreted, Wnt7a-specific inhibitors can terminate osteogenic differentiation. Further investigations are required to explore Wnt7a inhibitors in vivo and in in vitro. The data presented herein provide an opportunity to explore the role of Wnt7a in pathogenic mechanisms of bone-related cancer and therapeutic intervention for bone damage repair.

\section{Acknowledgements}

Not applicable.

\section{Funding}

The present study was supported by funding from the National Natural Science Foundation of China (no. 81860516), the start-up grant from the Affiliated Hospital of Guizhou Medical University (no. I201802), Guizhou science and Technology Department (no. 2016J7234), Guizhou science and Technology Department (no. 2014LH7149), the National Natural Science Foundation of China (no. 81460448), the R\&D infrastructure and facility Development and Program of Guizhou (no. 20154005).

\section{Availability of data and materials}

The datasets used and/or analyzed during the current study are available from the corresponding author on reasonable request.

\section{Authors' contributions}

LZ, PH and XD conceived and designed the experiments. LY, QL, JZ, PL, CW, PA, CW and XZ performed the experiments. $\mathrm{XD}$ analyzed the data. $\mathrm{LZ}, \mathrm{PH}$ and XD wrote the manuscript. LY, XD and LZ confirm the authenticity of all the raw data. All authors reviewed the paper and approved the final manuscript.

\section{Ethics approval and consent to participate}

All experiments and protocols were approved by the Ethics Committee of Guizhou Medical University. Each patient provided written informed consent prior to the preparation of the bone tissues.

\section{Patient consent for publication}

Not applicable.

\section{Competing interests}

The authors declare that they have no competing interests.

\section{References}

1. Pittenger MF, Mackay AM, Beck SC, Jaiswal RK, Douglas R, Mosca JD, Moorman MA, Simonetti DW, Craig S and Marshak DR: Multilineage potential of adult human mesenchymal stem cells. Science 284: 143-147, 1999.

2. Lin RZ, Moreno-Luna R, Li D, Jaminet SC, Greene AK and Melero-Martin JM: Human endothelial colony-forming cells serve as trophic mediators for mesenchymal stem cell engraftment via paracrine signaling. Proc Natl Acad Sci USA 111: 10137-10142, 2014.

3. Caplan AI: Mesenchymal stem cells. J Orthop Res 9: 641-650, 1991.

4. Caplan AI and Bruder SP: Mesenchymal stem cells: Building blocks for molecular medicine in the 21st century. Trends Mol Med 7: 259-264, 2001

5. Yang N, Wang G, Hu C, Shi Y, Liao L, Shi S, Cai Y, Cheng S, Wang X, Liu Y, et al: Tumor necrosis factor $\alpha$ suppresses the mesenchymal stem cell osteogenesis promoter miR-21 in estrogen deficiency-induced osteoporosis. J Bone Miner Res 28: 559-573, 2013.

6. Alford AI, Kozloff KM and Hankenson KD: Extracellular matrix networks in bone remodeling. Int J Biochem Cell Biol 65: 20-31, 2015.

7. Beck GR Jr, Khazai NB, Bouloux GF, Camalier CE, Lin Y, Garneys LM, Siqueira J, Peng L, Pasquel F, Umpierrez D, et al: The effects of thiazolidinediones on human bone marrow stromal cell differentiation in vitro and in thiazolidinedione-treated patients with type 2 diabetes. Transl Res 161: 145-155, 2013.

8. Tang Y, Xie H, Chen J, Geng L, Chen H, Li X, Hou Y, Lu L, Shi S, Zeng $\mathrm{X}$ and Sun L: Activated NF- $\mathrm{kB}$ in bone marrow mesenchymal stem cells from systemic lupus erythematosus patients inhibits osteogenic differentiation through downregulating Smad signaling. Stem Cells Dev 22: 668-678, 2012.

9. D'Amelio P, Tamone C, Sassi F, D'Amico L, Roato I, Patanè S Ravazzoli M, Veneziano L, Ferracini R, Pescarmona GP and Isaia GC: Teriparatide increases the maturation of circulating osteoblast precursors. Osteoporos Int 23: 1245-1253, 2012.

10. Zhang S, Chen X, Hu Y, Wu J, Cao Q, Chen S and Gao Y: All-trans retinoic acid modulates Wnt $3 \mathrm{~A}$-induced osteogenic differentiation of mesenchymal stem cells via activating the PI3K/AKT/GSK3 $\beta$ signalling pathway. Mol Cell Endocrinol 422: 243-253, 2016

11. Noronha-Matos JB and Correia-de-Sá P: Mesenchymal stem cells ageing: Targeting the 'Purinome' to promote osteogenic differentiation and bone repair. J Cell Physiol 231: 1852-1861, 2016.

12. Liu H, Xia X and Li B: Mesenchymal stem cell aging: Mechanisms and influences on skeletal and non-skeletal tissues. Exp Biol Med (Maywood) 240: 1099-1106, 2015. 
13. Anastas JN and Moon RT: WNT signalling pathways as therapeutic targets in cancer. Nat Rev Cancer 13: 11-26, 2013.

14. Zhou L and Liu Y: Wnt/beta-catenin signalling and podocyte dysfunction in proteinuric kidney disease. Nat Rev Nephrol 11: 535-545, 2015.

15. Hynes NE, Ingham PW, Lim WA, Marshall CJ, Massagué J and Pawson T: Signalling change: Signal transduction through the decades. Nat Rev Mol Cell Biol 14: 393-398, 2013.

16. Clevers $\mathrm{H}$ and Nusse $\mathrm{R}: \mathrm{Wnt} / \beta$-catenin signaling and disease. Cell 149: 1192-1205, 2012.

17. Palomo T, Al-Jallad H, Moffatt P, Glorieux FH, Lentle B, Roschger P, Klaushofer K and Rauch F: Skeletal characteristics associated with homozygous and heterozygous WNT1 mutations. Bone 67: 63-70, 2014

18. Laine CM, Joeng KS, Campeau PM, Kiviranta R, Tarkkonen K, Grover M, Lu JT, Pekkinen M, Wessman M, Heino TJ, et al: WNT1 mutations in early-onset osteoporosis and osteogenesis imperfecta. N Engl J Med 368: 1809-1816, 2013.

19. Rauch F and Glorieux FH: Osteogenesis imperfecta. Lancet 363: 1377-1385, 2004.

20. Keupp K, Beleggia F, Kayserili H, Barnes AM, Steiner M, Semler O, Fischer B, Yigit G, Janda CY, Becker J, et al: Mutations in WNT1 cause different forms of bone fragility. Am J Hum Genet 92: 565-574, 2013.

21. Keller KC, Ding H, Tieu R, Sparks NR, Ehnes DD and Zur Nieden NI: Wnt5a supports osteogenic lineage decisions in embryonic stem cells. Stem Cells Dev 25: 1020-1032, 2016.

22. Chen J, Tu X, Esen E, Joeng KS, Lin C, Arbeit JM, Rüegg MA, Hall MN, Ma L and Long F: WNT7B promotes bone formation in part through mTORC1. PLoS Genet 10: e1004145, 2014.

23. Tan SH, Senarath-Yapa K, Chung MT, Longaker MT, Wu JY and Nusse R: Wnts produced by Osterix-expressing osteolineage cells regulate their proliferation and differentiation. Proc Natl Acad Sci USA 111: E5262-E5271, 2014

24. Cawthorn WP, Bree AJ, Yao Y, Du B, Hemati N, Martinez-Santibañez G and MacDougald OA: Wnt6, Wnt10a and Wnt10b inhibit adipogenesis and stimulate osteoblastogenesis through a $\beta$-catenin-dependent mechanism. Bone 50: 477-489, 2012

25. Cheng SL, Shao JS, Cai J, Sierra OL and Towler DA: Msx2 exerts bone anabolism via canonical Wnt signaling. J Biol Chem 283 20505-20522, 2008

26. Ye L, Fan Z, Yu B, Chang J, Al Hezaimi K, Zhou X, Park NH and Wang CY: Histone demethylases KDM4B and KDM6B promotes osteogenic differentiation of human MSCs. Cell Stem Cell 11: 50-61, 2012.

27. Najdi R, Proffitt K, Sprowl S, Kaur S, Yu J, Covey TM, Virshup DM and Waterman ML: A uniform human Wnt expression library reveals a shared secretory pathway and unique signaling activities. Differentiation 84: 203-213, 2012

28. Cao Y, Sun Z, Liao L, Meng Y, Han Q and Zhao RC: Human adipose tissue-derived stem cells differentiate into endothelial cells in vitro and improve postnatal neovascularization in vivo. Biochem Biophys Res Commun 332: 370-379, 2005.

29. Livak KJ and Schmittgen TD: Analysis of relative gene expression data using real-time quantitative PCR and the 2(-Delta Delta C(T)) method. Methods 25: 402-408, 2001

30. Dou XW, Liang YK, Lin HY, Wei XL, Zhang YQ, Bai JW, Chen CF, Chen M, Du CW, Li YC, et al: Notch3 maintains luminal phenotype and suppresses tumorigenesis and metastasis of breast cancer via trans-activating estrogen receptor- $\alpha$. Theranostics 7: 4041-4056, 2017.

31. Oosterwegel MA, van de Wetering ML, Holstege FC, Prosser HM, Owen MJ and Clevers HC: TCF-1, a T cell-specific transcription factor of the HMG box family, interacts with sequence motifs in the TCR $\beta$ and TCR $\delta$ enhancers. Int Immunol 3: 1189-1192, 1991
32. van Beest M, Dooijes D, van De Wetering M, Kjaerulff S, Bonvin A, Nielsen O and Clevers H: Sequence-specific high mobility group box factors recognize 10-12-base pair minor groove motifs. J Biol Chem 275: 27266-27273, 2000.

33. Gaur T, Lengner CJ, Hovhannisyan H, Bhat RA, Bodine PV, Komm BS, Javed A, van Wijnen AJ, Stein JL, Stein GS and Lian JB: Canonical WNT signaling promotes osteogenesis by directly stimulating Runx2 gene expression. J Biol Chem 280: 33132-33140, 2005.

34. Tseng PC, Hou SM, Chen RJ, Peng HW, Hsieh CF, Kuo ML and Yen ML: Resveratrol promotes osteogenesis of human mesenchymal stem cells by upregulating RUNX2 gene expression via the SIRT1/FOXO3A axis. J Bone Miner Res 26: 2552-2563, 2011.

35. Baglìo SR, Devescovi V, Granchi D and Baldini N: MicroRNA expression profiling of human bone marrow mesenchymal stem cells during osteogenic differentiation reveals Osterix regulation by miR-31. Gene 527: 321-331, 2013.

36. Li E, Zhang J, Yuan T and Ma B: MiR-143 suppresses osteogenic differentiation by targeting Osterix. Mol Cell Biochem 390: 69-74, 2014.

37. AlAmer N, Bondalapati A, Garcia-Godoy F and Kandalam U: Osteogenic differentiation of orofacial tissue-derived mesenchymal stem cells-A review. Curr Tissue Eng 5: 11-20, 2016.

38. Atashi F, Modarressi A and Pepper MS: The role of reactive oxygen species in mesenchymal stem cell adipogenic and osteogenic differentiation: A review. Stem Cells Dev 24: 1150-1163, 2015.

39. Fakhry M, Hamade E, Badran B, Buchet R and Magne D: Molecular mechanisms of mesenchymal stem cell differentiation towards osteoblasts. World J Stem Cells 5: 136-148, 2013.

40. Deng ZL, Sharff KA, Tang N, Song WX, Luo J, Luo X, Chen J, Bennett E, Reid R, Manning D, et al: Regulation of osteogenic differentiation during skeletal development. Front Biosci 13: 2001-2021, 2008.

41. Harada SI and Rodan GA: Control of osteoblast function and regulation of bone mass. Nature 423: 349-355, 2003.

42. Komori T: Regulation of bone development and maintenance by Runx2. Front Biosci 13: 898-903, 2007.

43. James AW: Review of signaling pathways governing MSC osteogenic and adipogenic differentiation. Scientifica (Cairo) 2013: 684736, 2013.

44. Nakashima K,Zhou X,Kunkel G,Zhang Z, Deng JM,Behringer RR and de Crombrugghe B: The novel zinc finger-containing transcription factor osterix is required for osteoblast differentiation and bone formation. Cell 108: 17-29, 2002.

45. Artigas N, Gámez B, Cubillos-Rojas M, Sánchez-de Diego C, Valer JA, Pons G, Rosa JL and Ventura F: p53 inhibits SP7/Osterix activity in the transcriptional program of osteoblast differentiation. Cell Death Differ 24: 2022-2031, 2017.

46. Leucht $P$ and Helms JA: Wnt signaling: An emerging target for bone regeneration. J Am Acad Orthop Surg 23: 67-68, 2015.

47. Yin X, Li J, Salmon B, Huang L, Lim WH, Liu B, Hunter DJ, Ransom RC, Singh G, Gillette M, et al: Wnt signaling and its contribution to craniofacial tissue homeostasis. J Dental Res 94: 1487-1494, 2015

48. van de Wetering M, Oosterwegel M, Dooijes D and Clevers H: Identification and cloning of TCF-1, a T lymphocyte-specific transcription factor containing a sequence-specific HMG box. EMBO J 10: 123-132, 1991.

This work is licensed under a Creative Commons Attribution-NonCommercial-NoDerivatives 4.0 International (CC BY-NC-ND 4.0) License. 